\title{
Dosimétrie cellulaire en médecine nucléaire diagnostique: influence des émissions électroniques
}

\author{
I. GARDIN*,**, M. FARAGGI*, J.-L. STIEVENART*, \\ D. LE GULUDEC* $*$ B. BOK*
}

(Manuscrit reçu le 8 janvier 1998, accepté le 30 mars 1998)

RÉSUMÉ Les radio-nucléides utilisés en diagnostic présentent des émissions photoniques utilisées pour l'imagerie, mais également des émissions électroniques de faible énergie, responsables d'une hétérogénéité de dose à l'échelon cellulaire. La dose moyenne délivrée au noyau par les émissions électroniques du ${ }^{99 \mathrm{~m}} \mathrm{Tc}$, de $\mathrm{l}^{123} \mathrm{I}$, de

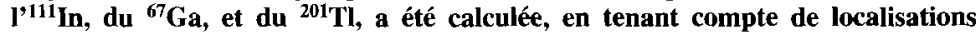
nucléaires, cytoplasmiques et membranaires de la radioactivité. Ce modèle tient compte de la contribution de la dose due à la radioactivité présente dans la cellule cible elle-même, et de la contribution des cellules voisines. Les résultats de la dosimétrie cellulaire ( $\left.D^{\text {cet }}\right)$ ont été comparés à ceux trouvés en dosimétrie conventionnelle ( $\left.D^{\mathrm{onv}}\right)$, en supposant une activité identique dans toutes les cellules. Le modèle cellulaire montre que pour des localisations membranaires et cytoplasmiques, la principale source d'irradiation électronique vient des cellules voisines. Pour des localisations nucléaires, la contribution de la cellule cible n'est plus négligeable et peut même devenir la principale contribution. La comparaison entre les modèles cellulaire et conventionnel montre que $D^{\mathrm{cel} /} D^{\mathrm{conv}}$ varie entre 0,61 et 0,89 pour des localisations cytoplasmiques et membranaires de la radioactivité, suivant le radionucléide et les dimensions cellulaires. Ainsi, la dosimétrie conventionnelle surestime faiblement la dose au noyau. Par contre, $D^{\mathrm{cel}} / D^{\mathrm{conv}}$ varie entre 1,1 et 75 pour une localisation purement nucléaire. La dosimétrie conventionnelle peut alors sous-estimer notablement la dose au noyau. Cette étude montre qu'en médecine nucléaire diagnostique, la dosimétrie cellulaire peut conduire à une radioprotection accrue du patient et une meilleure évaluation des risques radiobiologiques liés à l'administration d'un radiopharmaceutique.

ABSTRACT Cellular dosimetry in nuclear medecine imaging: influence of electron emissions The radionuclides used in nuclear medicine imaging emit not only diagnostically useful photons, but also low energy electron emissions, responsible for dose heterogeneity at the cellular level. The mean dose delivered to the cell nucleus by electron emissions of ${ }^{99 \mathrm{~m}} \mathrm{Tc},{ }^{123} \mathrm{I},{ }^{111} \mathrm{In},{ }^{67} \mathrm{Ga}$, and ${ }^{201} \mathrm{Tl}$, has been calculated, for a cell nucleus, a cytoplasmic and a cell membrane distribution of radioactivity. This model

\footnotetext{
Unité de recherche Chimie et Biophysique des Traceurs, Université X. Bichat. Paris 75018 France.

** Mél : isabelle.gardin@bjn.ap-hop-paris.fr
} 


\begin{abstract}
takes into account both the self-dose which results from the radionuclide located in the target cell, and the cross-dose, which comes from the surrounding cells. The results obtained by cellular dosimetry $\left(D^{\text {cel }}\right)$ have been compared with those obtained with conventional dosimetry $\left(D^{\text {conv }}\right)$, by assuming the same amount of radioactivity per cell. Cellular dosimetry shows, for a cytoplasmic and a cell membrane distributions of radioactivity, that the main contribution to the dose to the cell nucleus, comes from the surrounding cells. On the other hand, for a cell nucleus distribution of radioactivity, the self-dose is not negligible and may be the main contribution. The comparison between cellular and conventional dosimetry shows that $D^{\mathrm{cel}} / D^{\mathrm{conv}}$ ratio ranges from 0.61 and 0.89 , in case of a cytoplasmic and a cell membrane distributions of radioactivity, depending on the radionuclide and cell dimensions. Thus, conventional dosimetry slightly overestimates the mean dose to the cell nucleus. On the other hand, $D^{\mathrm{cel} /} / D^{\mathrm{con}}$ ranges from 1.1 to 75 , in case of a cell nucleus distribution of radioactivity. Conventional dosimetry may strongly underestimates the absorbed dose to the nucleus, when radioactivity is located in the nucleus. This study indicates that in nuclear medicine imaging, cellular dosimetry may lead to a better understanding of biological effects of radiopharmaceuticals.
\end{abstract}

\title{
1. Introduction
}

Le principe de la médecine nucléaire diagnostique repose sur l'administration, généralement par voie intraveineuse, d'une molécule marquée au patient (ou radiopharmaceutique). Les radio-nucléides utilisés présentent des émissions photoniques détectés par une gamma-caméra. Le but est d'obtenir des images de la répartition in-vivo du radio-traceur.

L'évaluation des risques radiobiologiques liés à cette administration, reposent sur le calcul de la dose moyenne reçue par les organes cibles. On parle alors de dosimétrie classique ou conventionnelle. Les calculs postulent que la répartition du radiopharmaceutique est uniforme au niveau des organes sources et que la distribution de la dose absorbée est homogène au niveau des organes cibles (Loevinger et al., 1988). Ces hypothèses permettent de simplifier la modélisation, et les données dosimétriques sont disponibles pour les radiopharmaceutiques utilisées en routine clinique (ICRP, 1992).

Ces hypothèses sont généralement vérifiées pour les émissions photoniques, car celles-ci ont un libre parcours moyen très supérieur à la taille des organes (Makrigiorgos et al., 1989). Cependant, la plupart des radio-nucléides utilisés en médecine nucléaire diagnostique présentent également des émissions électroniques de faible énergie. Ces électrons ont un parcours très limité dans les tissus biologiques, et peuvent être responsables d'une hétérogénéité de dose à l'échelon cellulaire (Faraggi et al., 1994 ; Humm et al., 1994).

Nous allons présenter les outils que nous avons développé pour effectuer une dosimétrie cellulaire, correspondant à l'irradiation des noyaux cellulaires par les émissions électroniques du ${ }^{99 \mathrm{~m}} \mathrm{Tc}$, de $1^{1}{ }^{123} \mathrm{I}$, de $\mathrm{l}^{1111} \mathrm{In}$, du ${ }^{67} \mathrm{Ga}$, et du ${ }^{201} \mathrm{Tl}$. Nous 
présenterons les principaux résultats que l'on peut tirer des simulations informatiques, ainsi que le domaine de validité de la dosimétrie classique. Le modèle cellulaire tient compte de la radioactivité localisée sur la cellule cible elle-même et sur les cellules voisines, suivant un empilement hexagonal compact.

\section{Matériel et Méthodes}

\subsection{Principe de la modélisation}

Le formalisme utilisé pour effectuer des calculs de dose à l'échelon cellulaire s'inscrit dans la méthodologie proposée par le MIRD Committee (Medical Internal Radiation Dose) (Loevinger et al., 1988). Suivant ce formalisme, la dose moyenne délivrée par les émissions électroniques qui est absorbée dans une région cible $\mathbf{r}_{\mathrm{k}}$ due à une activité cumulée $\tilde{A}_{\mathrm{h}}$ dans une région source $\mathbf{r}_{\mathrm{h}}$, vérifie la relation :

$$
\mathrm{D}\left(\mathbf{r}_{\mathrm{k}} \leftarrow \mathbf{r}_{\mathrm{h}}\right)=\tilde{A_{\mathrm{h}}} \times S\left(\mathbf{r}_{\mathrm{k}} \leftarrow \mathbf{r}_{\mathrm{h}}\right)
$$

où $S$ représente la dose absorbée moyenne par unité d'activité cumulée :

$$
S\left(\mathbf{r}_{\mathrm{k}} \leftarrow \mathbf{r}_{\mathrm{h}}\right)=K \frac{\sum_{i} n_{i} E_{i} \Phi_{i}\left(\mathbf{r}_{\mathrm{k}} \leftarrow \mathbf{r}_{\mathrm{h}}\right)}{m_{k}}
$$

$m_{\mathrm{k}}$ est la masse de la cible, $n_{i}$ est le nombre d'électrons d'énergie $E_{i}$ émis par désintégration. $\Phi_{i}$ représente la fraction de l'énergie $E_{i}$ émise par la source qui est absorbée dans la cible. $K$ est une constante qui dépend des unités choisies, dont la valeur sera précisée plus loin.

Avec ce formalisme, aucune hypothèse n'est faite sur la taille et la nature de la source et de la cible. Si en dosimétrie conventionnelle la cible et la source sont des organes, en dosimétrie cellulaire la cible et la source sont des cellules et des compartiments cellulaires (Howell, 1994).

\subsection{Sources et cibles en dosimétrie cellulaire}

En raison de la présence d'ADN, le noyau est généralement considéré comme la cible cellulaire (Hofer et al., 1975). Lorsque la source et la cible sont confondues, on parle de dose auto-absorbée par la cellule elle-même ( $D_{\text {self }}^{\text {cel }}$ (noyau $\leftarrow \mathbf{r}_{\mathbf{h}}$ ) ou self-dose). Lorsque la cible et les sources sont différentes, on parle de contribution des cellules 
voisines sur la dose au noyau d'une cellule cible centrale ( $D_{\text {cross }}^{\text {cel }}$ (noyau $\leftarrow \mathbf{r}_{\mathrm{h}}$ ) ou cross-dose) (Goddu et al., 1994). On a alors la somme de deux termes pour calculer la dose moyenne au noyau cellulaire :

$$
D^{\text {cel }}\left(\text { noyau } \leftarrow \mathbf{r}_{\mathrm{h}}\right)=D_{\text {self }}^{\text {cel }}\left(\text { noyau } \leftarrow \mathbf{r}_{\mathbf{h}}\right)+D_{\text {cross }}^{\text {cel }}\left(\text { noyau } \leftarrow \mathbf{r}_{\mathrm{h}}\right)
$$

avec par exemple pour la contribution de la dose auto-absorbée par la cellule :

$$
D_{\text {self }}^{\text {cel }}\left(\text { noyau } \leftarrow \mathbf{r}_{\mathrm{h}}\right)=\tilde{\mathrm{A}}_{\mathrm{h}-\text { self }} \times S_{\text {self }}^{\text {cel }}\left(\text { noyau } \leftarrow \mathbf{r}_{\mathrm{h}}\right)
$$

où $\tilde{A}_{\mathrm{h} \text {-self }}$ représente l'activité cumulée sur la cellule cible et $S_{\text {cross }}^{\mathrm{cel}}\left(\right.$ noyau $\leftarrow \mathbf{r}_{\mathrm{h}}$ ) vérifie :

$$
D_{\text {self }}^{\text {cel }}\left(\text { noyau } \leftarrow \mathbf{r}_{\mathrm{h}}\right)=K \frac{\sum_{i} n_{i} E_{i} \Phi_{i-\text { self }}^{\text {cel }}\left(\text { noyau } \leftarrow \mathbf{r}_{\mathrm{h}}\right)}{m_{\text {noyau }}}
$$

$\Phi_{\mathrm{i} \text {-self }}^{\text {cel }}$ (noyau $\leftarrow \mathbf{r}_{\mathrm{h}}$ ) représente la fraction de l'énergie $E_{i}$ émise dans la cellule cible qui est absorbée dans son noyau, $m_{\text {noyau }}$ est la masse du noyau cible. Si l'on considère les cellules comme sphériques, de noyau centré, et de densité unité, $S_{\text {self }}^{\text {cel }}\left(\right.$ noyau $\left.\leftarrow \mathbf{r}_{\mathrm{h}}\right)\left(\right.$ en $\left.\mathrm{mGy} \mathrm{Bq} \mathrm{keV} \mathrm{B}^{-1}\right)$ peut s'exprimer en fonction du rayon nucléaire $R$ noyau (en $\mu \mathrm{m})$ :

$$
S_{\text {self }}^{\text {cel }}\left(\text { noyau } \leftarrow \mathbf{r}_{\mathbf{h}}\right)=K \frac{\sum_{\mathrm{i}} n_{i} E_{i} \Phi_{i-\text { self }}^{\text {cel }}\left(\text { noyau } \leftarrow \mathbf{r}_{\mathrm{h}}\right)}{R_{\text {noyau }}^{3}}
$$

Notons que l'énergie absorbée dans le noyau cellulaire vérifie également la relation suivante:

$$
\bar{\varepsilon}_{\text {self }}\left(\text { noyau } \leftarrow \mathbf{r}_{\mathbf{h}}\right)=\sum_{i} n_{i} E_{i} \Phi_{i-\text { self }}^{\text {cel }}\left(\text { noyau } \leftarrow \mathbf{r}_{\mathrm{h}}\right) .
$$

Le même type de relations existe pour la contribution des cellules voisines ( $S_{\text {cross }}^{\text {eel }}$ et $\overline{\boldsymbol{\varepsilon}}_{\text {cross }}$ ). Les paramètres qui interviennent sont alors : $\tilde{A}_{\text {h-cross }}$ l'activité cumulée sur les cellules avoisinantes, et $\Phi_{i \text {-cross }}^{\text {cel }}$ la fraction de l'énergie émise par les cellules proches qui est absorbée dans le noyau de la cellule cible centrale.

Le problème mathématique réside dans la détermination de $\Phi_{i \text {-self }}^{\text {cel }}$ et $\Phi_{i \text {-cross }}^{\text {cel }}$, pour chaque valeur d'énergie $E_{i}$, et chaque localisation sub-cellulaire de la radioactivité. 


\subsection{Modèle cellulaire}

Plusieurs localisations intracellulaires $\left(\mathbf{r}_{h}\right)$ de la radioactivité ont été étudiées : des localisations nucléaires, cytoplasmiques ou membranaires. La simulation a porté sur des cellules de rayon $\left(R_{\text {cel }}\right)$ variant entre 3 et $12 \mu \mathrm{m}$ et de rayon nucléaire $\left(R_{\text {noyau }}\right)$ variant de 2 à $11 \mu \mathrm{m}$.

Pour le calcul de $S_{\text {cross }}^{\mathrm{cel}}$, les cellules sont supposées être agencées suivant un empilement hexagonal compact. Le nombre de cellules participant à $S_{\text {cross }}^{\mathrm{cel}}$, dépend des dimensions cellulaires et de l'énergie d'émission de l'électron. La probabilité qu'un électron dépose de l'énergie à une distance supérieure à son parcours moyen n'est pas totalement nulle. Ainsi, toute cellule située à une distance inférieure à 1,2 fois le parcours moyen de l'électron est susceptible de déposer de l'énergie à la cellule cible centrale. La contribution de chacune de ces cellules a été calculée et additionnée pour déterminer $S_{\text {cross }}^{\mathrm{cel}}$. Le modèle suppose une activité cumulée identique sur toutes les cellules.

\subsection{Modélisation de la fraction absorbée en dosimétrie cellulaire}

Pour chaque contribution ( $D_{\text {self }}^{\text {cel }}$ et $D_{\text {cross }}^{\text {cel }}$ ), le calcul des différentes fractions absorbées $\Phi_{i}^{\text {cel }}$, comporte deux étapes. Dans un premier temps est calculé pour chaque énergie $E_{i}$ : le parcours maximal $r i$ de l'électron, la fonction $F\left(\frac{r}{r_{i}}, E_{i}\right)$ modélisant le dépôt d'énergie de l'électron le long de son parcours dans le modèle de la source ponctuelle. Puis, dans un second temps, pour prendre en compte les différentes distributions géométriques des sources (nucléaire, cytoplasmique, membranaire), nous avons calculé la contribution de chaque point source à la dose absorbée au noyau cellulaire.

Les calculs de $r_{i}$ et de $F\left(\frac{r}{r_{i}}, E_{i}\right)$ reposent sur la modélisation analytique proposée par Prewich (Prestwich et al., 1985) et Kwok (Kwok et al., 1987). Pour la prise en compte des distributions volumiques de la radioactivité, nous avons utilisé des expressions mathématiques déjà publiées pour le calcul de $S_{\text {self }}^{\mathrm{cel}}$ (Gardin $e t$ al., 1995), et celles proposées par Goddu pour $S_{\text {cross }}^{\text {cel }}$ (Goddu et al., 1994).

\subsection{Radionucléides}

Ce travail a été réalisé avec les radioéléments couramment utilisés en médecine nucléaire diagnostique, soit : le ${ }^{99 \mathrm{~m}} \mathrm{Tc}, \mathrm{l}^{123} \mathrm{I}, \mathrm{l}^{111} \mathrm{In}, \mathrm{le}{ }^{67} \mathrm{Ga}$, et le ${ }^{201} \mathrm{Tl}$. Les spectres des émissions électroniques de ces radio-nucléides sont reportés dans le tableau I d'après les données de Howell (Howell, 1992). 


\section{TABLEAU I}

Spectres des émissions électroniques d'après Howell (Howell, 1994).

$E_{1}$ énergie en keV, $n_{1}$ abondance de l'émission.

Electron radiation spectra from Howell (Howell, 1994). $E_{i}$ energy in keV and $n_{i}$ yield per decay.

\begin{tabular}{|c|c|c|c|c|c|c|c|c|c|}
\hline \multicolumn{4}{|c|}{ 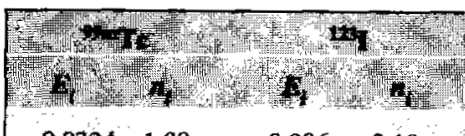 } & 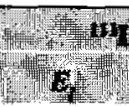 & \multicolumn{4}{|c|}{ 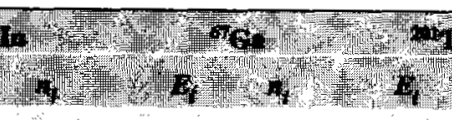 } & 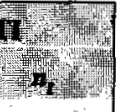 \\
\hline 0,0334 & 1,98 & 0,006 & 2,18 & 0,00847 & 7,82 & 0,0624 & 2,07 & 0,0161 & 17,6 \\
\hline 0,0429 & 0,0193 & 0,0298 & 2,10 & 0,0388 & 2,54 & 0,0729 & 0,346 & 0,0453 & 2,84 \\
\hline 0,116 & 0,747 & 0,0325 & 6,54 & 0,125 & 0,915 & 0,921 & 1,68 & 0,0644 & 7,93 \\
\hline 0,226 & 1,10 & 0,127 & 0,869 & 0,183 & 0,151 & 0,953 & 0,0116 & 0,172 & 4,41 \\
\hline 1,82 & 0,991 & 0,213 & 0,156 & 0,350 & 2,09 & 7,43 & 0,470 & 0,406 & 0,923 \\
\hline 2,05 & 0,0868 & 0,461 & 1,97 & $\therefore \quad 2,59$ & 0,835 & 8,44 & 0,116 & 0,773 & 0,322 \\
\hline 2,32 & 0,0137 & 3,04 & 0,751 & 3,06 & 0,190 & 9,46 & 0,0082 & 0,895 & 0,608 \\
\hline 2,66 & 0,0012 & 3,66 & 0,202 & 3,53 & 0,0109 & 81,6 & 0,0027 & 1,83 & $2 ; 03$ \\
\hline 15,3 & 0,0126 & 4,28 & 0,013 & 19,1 & 0,103 & 83,7 & 0,270 & 7,58 & 0,541 \\
\hline 17,8 & 0,0047 & 22,4 & 0,0838 & 22,3 & 0,0394 & 92,2 & 0,0376 & 9,85 & 0,235 \\
\hline 119 & 0,0843 & 26,3 & 0,0384 & 25,5 & 0,0036 & 93,2 & 0,0066 & 12,0 & 0,0191 \\
\hline 122 & 0,0059 & 30,2 & 0,0035 & 145 & 0,0824 & 175 & 0,0034 & 12,2 & 0,0022 \\
\hline 137 & 0,0136 & 127 & 0,130 & 167 & 0,01 & 291 & $0, \infty 01$ & 15,9 & 0,0861 \\
\hline 140 & 0,0062 & 154 & 0,0179 & 171 & 0,0014 & & & 17,4 & 0,0724 \\
\hline & & 158 & 0.0053 & 219 & 0,0521 & & & 27.7 & 0,0236 \\
\hline & & & & 241 & 0,0091 & & & 29.4 & 0,0237 \\
\hline & & & & 245 & 0,0019 & & & 52,2 & 0,0797 \\
\hline & & & & & & & & 55,0 & 0,0268 \\
\hline & & & & & & & & 66,3 & 0,0153 \\
\hline & & & & & & & : & 77,5 & 0,0015 \\
\hline & $\therefore$ & & & & & & & 82,8 & 0,0025 \\
\hline & & & & & & & & 84,3 & 0,159 \\
\hline & & & & & & & & 121 & 0,0152 \\
\hline " & & & & & & & & 133 & 0,0027 \\
\hline & & & & & & & & 153 & 0,0269 \\
\hline & & & & & & & & 165 & 0,0094 \\
\hline
\end{tabular}




\subsection{Comparaison entre dosimétrie cellulaire et conventionnelle}

En dosimétrie conventionnelle, la distribution de dose est supposée homogène sur tout l'organe cible. Ceci signifie que l'on considère qu'il existe un équilibre électronique dans le volume que constitue l'organe. Quel que soit le volume étudié, la quantité d'énergie absorbée dans ce volume correspond à la quantité d'energie produite dans ce volume. Ainsi par exemple, l'énergie absorbée dans le volume occupé par la cellule $\left(\bar{\varepsilon}_{\text {tot }}\right)$, correspond à l'énergie électronique totale $\left(E_{\text {tot }}\right)$, émise par cette cellule : $\bar{\varepsilon}_{\text {tot }}=E_{\text {tot }}$. Dans le cas d'un empilement hexagonal compact, cet espace est 1,35 fois le volume de la cellule.

Par contre dans le modèle cellulaire, seule une fraction de l'énergie émise est absorbée dans le noyau, cette fraction correspond à la contribution du radiotraceur sur la cellule elle-même. Pour une même activité par cellule, on a :

$$
\frac{D^{\text {cel }}}{D^{\text {conv }}}=1,35\left(\frac{R_{\text {cel }}}{R_{\text {noyau }}}\right)^{3}\left(\frac{\bar{\varepsilon}_{\text {self }}+\bar{\varepsilon}_{\text {cross }}}{\bar{\varepsilon}_{\text {tot }}}\right)
$$

\section{TABLEAU II}

Spectres des émissions électroniques du ${ }^{99 m}$ Tc d'après Howell (Howell, 1994), et parcours moyen $r_{i}$ correspondant.

Radiation spectrum of ${ }^{99 m}$ Tc electron emissions from Howell (Howell, 1994), and $r_{i}$ the corresponding range.

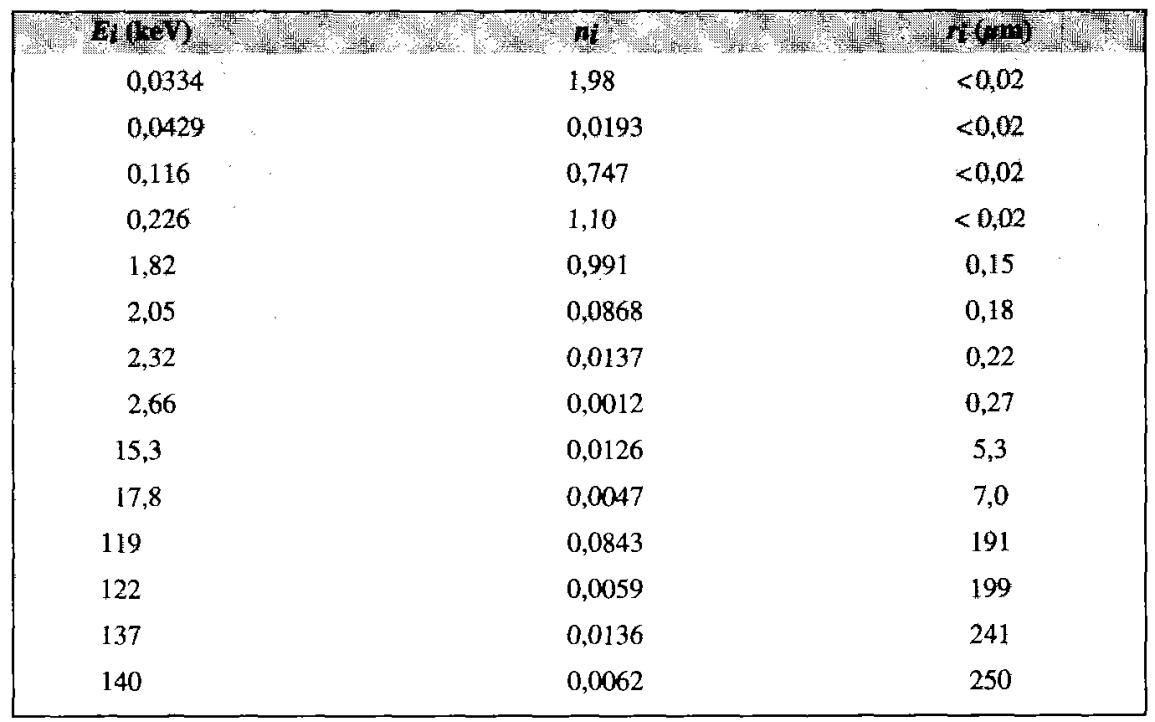




\section{Résultats}

Dans le tableau II est reporté le spectre des émissions électroniques du ${ }^{99 \mathrm{~m}} \mathrm{Tc}$ proposé par Howell (Howell, 1992), ainsi que les parcours moyens correspondants suivant notre modélisation. Seul le parcours moyen des émissions électroniques du ${ }^{99 m} \mathrm{mc}$ a été présenté dans ce tableau. Les autres radio-nucléides étudiés présentent également un spectre d'émission d'électrons mono-énergétiques s'étendant entre quelques centaines de $\mathrm{keV}$ à des valeurs inférieures au $\mathrm{keV}$, comme l'indique le tableau I, avec des parcours moyens s'étendant de quelques centaines de micromètre à des distances inférieures à $0,02 \mu \mathrm{m}$.

\section{TABLEAU III}

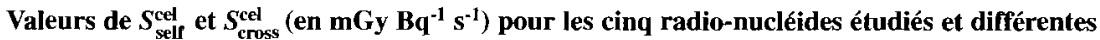
localisations subcellaires du radiopharmaceutique.

$S_{\text {self }}^{\text {cel }} S_{\text {cross }}^{\text {cel }}$ représentent les doses moyennes

par unité d'activité cumulée, délivrées au noyau cellulaire par les émissions électroniques.

$S_{\text {celf }}^{c e l}$ correspond à la contribution de la radioactivité sur la cible elle-même.

$S_{\text {cross }}^{\text {celf }}$ correspond à la contribution de la radioactivité sur les cellules voisines.

a) $R_{\text {noyau }}=4 \mu \mathrm{m}$ et $R_{\text {cet }}=8 \mu \mathrm{m}$.
b) $R_{\text {noyau }}=4 \mu \mathrm{m}$ et $R_{\text {cel }}=12 \mu \mathrm{m}$.

$S_{\text {self }}^{\text {cel }}$ and $S_{\text {cross }}^{\text {cel }}$ values (in $\mathrm{mGy} \mathrm{Bq}^{-1} \mathrm{~s}^{-1}$ ) for the five radionuclides studied and various subcellular localizations of radiopharmaceutical. $S_{\text {self }}^{\text {cel }}$ - and $S_{\text {cross }}^{\text {cel }}$ values represent the mean

dose per unit of cumulated activity, delivered to the cell nucleus by electron emissions.

$S_{\text {self }}^{\text {cel }}$ results from the radionuclide localized in the same cell, as $S_{\text {self }}^{\mathrm{cel}}$ comes from the radiation emanating from all other cell.

a) $R_{\text {nucleus }}=4 \mu \mathrm{m}$ and $R_{\text {cell }}=8 \mu \mathrm{m}$.

b) $R_{\text {nucleus }}^{\text {nucleus }}=4 \mu \mathrm{m}$ and $R_{\text {cell }}^{\text {cell }}=12 \mu \mathrm{m}$.

\begin{tabular}{|c|c|c|c|c|}
\hline \begin{tabular}{|l|l} 
a) \\
and
\end{tabular} & Wrat & 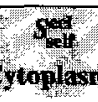 & 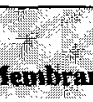 & 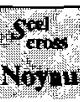 \\
\hline $99 \mathrm{~m} \mathrm{Tc}$ & 1,58 & 0,0303 & 0,0101 & 0,730 \\
\hline${ }^{123} \mathrm{I}$ & 3,01 & 0,146 & 0,0765 & 1,10 \\
\hline${ }^{111} \mathrm{In}_{\mathrm{In}}$ & 2,85 & 0,161 & 0,0641 & 1,53 \\
\hline${ }^{67} \mathrm{Ga}$ & 3,61 & 0,121 & 0,0334 & 1,47 \\
\hline${ }^{20} \mathrm{~F} \mathrm{Tl}$ & 8,24 & 0,332 & 0,0797 & 1,51 \\
\hline
\end{tabular}

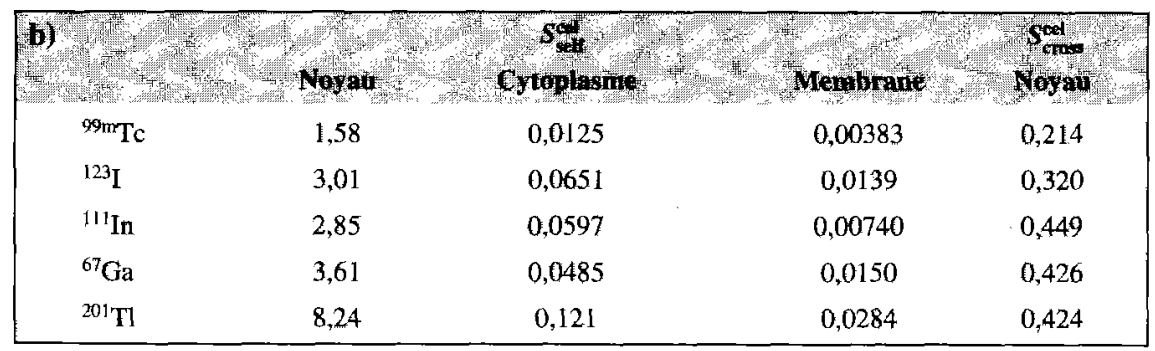


Dans les tableaux IIIa et IIIb sont reportées les valeurs de $S_{\text {self }}^{\mathrm{cel}}$ et $S_{\text {cross }}^{\mathrm{cel}}$ pour les cinq radio-nucléides étudiés, et des valeurs particulières de $R_{\text {noyau }}$ et $R_{\text {cel }}\left(R_{\text {noyau }}=\right.$ $4 \mu \mathrm{m}, R_{\text {cel }}=8 \mu \mathrm{m}$, Tab. IIIa et $R_{\text {noyau }}=4 \mu \mathrm{m}, R_{\text {cel }}=12 \mu \mathrm{m}$, tableau IIIb). Pour $S^{\text {ce }}$ self les valeurs correspondants aux trois localisations de la radioactivité sont données. Pour $S_{\text {cross }}^{\text {cel }}$, seuls les valeurs pour une localisation nucléaire sont présentées. Ceci tient au fait, que $S_{\text {cross }}^{\text {cel }}$ dépend peu de la localisation du traceur (variations inférieures à $5 \%$ pour l'ensemble des radio-nucléides et dimensions cellulaires étudiées), alors que $S_{\text {self }}^{\text {cel }}$ dépend très largement de la localisation intracellulaire du radiopharmaceutique. Pour l'ensemble des radio-nucléides et des dimensions cellulaires étudiées, les données numériques montrent que :

- pour des localisations membranaires et cytoplasmiques, la principale source d'irradiation électronique vient des cellules voisines,

- pour des localisations nucléaires, la dose auto-absorbée par la cellule ellemême n'est plus négligeable, et peut devenir la principale source d'irradiation.

\section{TABLEAU IV}

Rapport $D^{\text {cel}} / D^{\text {conv }}$ de la dose moyenne au noyau cellulaire délivrée par les émissions électroniques suivant une modélisation cellulaire (cel) et conventionnelle (conv). La simulation a porté sur trois localisations du radiotraceur (nucléaire, cycloplasmique et membranaire).

a) $R_{\text {noyau }}=4 \mu \mathrm{m}$ et $R_{\text {cel }}=8 \mu \mathrm{m}$.

b) $R_{\text {noyau }}=4 \mu \mathrm{m}$ et $R_{\text {cel }}=12 \mu \mathrm{m}$.

Ratio $D^{\mathrm{cH} /} / D^{\mathrm{conv}}$ of cellular (cel) to conventional (conv) electron dosimetry for three localizations of radioactivity (cell nucleus, cytoplasm and cell membrane).

a) $R_{\text {nucleus }}=4 \mu \mathrm{m}$ and $R_{\text {cel }}=8 \mu \mathrm{m}$.
b) $R_{\text {nucleus }}=4 \mu \mathrm{m}$ and $R_{\text {cell }}=12 \mu \mathrm{m}$.

\begin{tabular}{|lccc|}
\hline a) & Noyau & Cytoplasme & Membrane \\
${ }^{99}{ }^{9{ }_{\mathrm{m}}} \mathrm{Tc}$ & 2,58 & 0,85 & 0,83 \\
${ }^{123} \mathrm{I}$ & 2,71 & 0,82 & 0,78 \\
${ }^{111} \mathrm{In}$ & 2,28 & 0,88 & 0,83 \\
${ }^{67} \mathrm{Ga}$ & 2,72 & 0,85 & 0,80 \\
${ }^{201} \mathrm{Tl}$ & 3,89 & 0,74 & 0,64 \\
\hline
\end{tabular}

\begin{tabular}{|cccc|}
\hline b) & Noyau & Cytoplasme & Menobrane \\
${ }^{99 \mathrm{~m}} \mathrm{Tc}$ & 6,77 & 0,85 & 0,82 \\
${ }^{123} \mathrm{I}$ & 7,40 & 0,86 & 0,74 \\
${ }^{11} \mathrm{I} \mathrm{In}$ & 5,79 & 0,89 & 0,80 \\
${ }^{67} \mathrm{Ga}$ & 7,28 & 0,86 & 0,81 \\
${ }^{201} \mathrm{Tl}$ & 11,66 & 0,73 & 0,61 \\
\hline
\end{tabular}


À titre d'exemple, sont reportées, dans les tableaux IVa et IVb, les valeurs du rapport $D^{\text {cel }} / D^{\text {conv }}$ pour les cinq radio-nucléides étudiés, et deux jeux de dimensions cellulaires $\left(R_{\text {noyau }}=4 \mu \mathrm{m}, R_{\text {cel }}=8 \mu \mathrm{m}\right.$, Tab. IVa et $R_{\text {noyau }}=4 \mu \mathrm{m}, R_{\text {cel }}=12 \mu \mathrm{m}$, Tab IVb). Un calcul exhaustif pour l'ensemble des radio-nucléides, des rayons cellulaires et nucléaires étudiés montre que :

- $D^{\text {cel }} / D^{\text {conv }}$ varie entre 0,61 et 0,89 pour des localisations cytoplasmiques et membranaires de la radioactivité,

$-D^{\text {cel }} / D^{\text {conv }}$ varie entre 1,1 et 75 pour une localisation purement nucléaire.

\section{Discussion}

En médecine nucléaire diagnostique, les radio-nucléides présentent des émissions photoniques à des fins d'imagerie, mais également des émissions d'électrons monoénergétiques. Le libre parcours moyen des photons est grand par rapport à la taille des organes. De ce fait, l'hypothèse faite en dosimétrie classique, d'une répartition de dose homogène au niveau des organes cibles est généralement vérifiée (Makrigiorgos et al., 1989). Par contre, les émissions électroniques ont un parcours faible par rapport aux tissus biologiques et aux dimensions cellulaires, comme l'indique le tableau II, dans le cas du ${ }^{99 \mathrm{~m}} \mathrm{Tc}$. Cette propriété peut conduire à des hétérogénéités de dose importantes à l'échelon cellulaire (Humm et al., 1994). De ce fait, nous avons développé une modélisation de la dose délivrée à l'échelon cellulaire, dans le but d'offrir des outils de calcul et pour connaître le domaine de validité du modèle conventionnel. Cette modélisation, limitée à la dose auto-absorbée par la cellule (Gardin et al., 1995), s'étend à la contribution des cellules voisines.

Le modèle utilisé pour effectuer les calculs dosimétriques à l'échelon cellulaire s'appuie sur le formalisme du MIRD, largement utilisé en dosimétrie conventionnelle (Loevinger et al., 1988). Ce modèle, qui relève de la physique classique, est indépendant de la taille et de la forme des sources et des cibles. Il peut tout aussi bien s'appliquer à des organes, qu'à des volumes de plus petites tailles, tels que des cellules (Howell, 1994). Les valeurs calculées portent alors sur des doses absorbées moyennes sur le volume cible considéré, ici le noyau cellulaire. La modélisation analytique utilisée pour calculer le parcours moyen des électrons, $S_{\text {self }}^{\mathrm{cel}}$ et $S_{\text {cross }}^{\text {cel }}$ a été précédemment validé (Faraggi et al., 1990 ; Gardin et al., 1995).

Plusieurs hypothèses sur le modèle cellulaire ont été faites. Nous avons supposé les cellules sphériques, de noyau centré, toutes de mêmes dimensions, de densité unité, suivant un empilement hexagonal compact. Ces hypothèses sont bien évidemment contestables en raison de la complexité des tissus et la diversité des cellules. Cependant, des hypothèses doivent être formulées pour effectuer des simulations. Les modèles d'empilement cellulaire souvent rencontrées dans les tissus 
biologiques semblent être le modèle hexagonal compact et cubique centré (Makrigiorgos et al., 1989). Certains auteurs (Nettleton et Lawson, 1996) ont également comparé les valeurs de $S$ suivant une modélisation cellulaire sphérique et ellipsoïdale. Ces auteurs trouvent des écarts minimes entre ces deux modélisations eut égard à d'autres types d'incertitude (écart maximal de 2,1\% trouvé dans le cas $\left.\mathrm{du}{ }^{201} \mathrm{Tl}\right)$. Ce résultat est intéressant en particulier pour les spermatozoïdes, cellules géminales de forme ellipsoïdale.

Les données biologiques ont montré que la répartition du radio-traceur n'est pas nécessairement homogène sur toute la cellule, mais peut se fixer de façon privilégiée dans un compartiment sub-cellulaire (Puncher et Blower, 1994) (noyau, cytoplasme, membrane). Ces trois localisations ont été simulées. Cependant, à partir des données sur les localisations nucléaires et cytoplasmiques, il est facile d'en déduire (par simple addition des doses) les données pour une localisation homogène sur toute la cellule ou encore pour des concentrations radioactives différentes suivant le compartiment cellulaire.

Les données du tableau III montrent que la dose moyenne au noyau due aux électrons émis dans la cellule dépend très étroitement de la localisation sub-cellulaire de la radioactivité. Ce résultat s'interprète aisément à la lumière du spectre d'émission électronique des radio-nucléides étudiés. En effet, les électrons d'énergie inférieure au keV déposent toute leur énergie sur place. Lorsque le radiopharmaceutique est localisé dans le noyau, la totalité de l'énergie émise par ces électrons contribuera à la dose au noyau. Par contre, lorsque la radioactivité est extérieure au noyau, ces électrons auront un parcours trop faible pour atteindre le noyau cellulaire. Ils ne contribueront pas à $S_{\text {self }}^{\text {cel }}$.

Les données biologiques montrent que le comportement radiobiologique des radio-nucléides émetteurs d'électrons Auger dépend de leur localisation sub-cellulaire (Sastry, 1992). Ainsi, par exemple, Rao et al. (Rao et al., 1988), ont étudié in-vivo, la radiotoxicité de l'oxine- ${ }^{111}$ In et du citrate- ${ }^{111}$ In, après injection intratesticulaire chez la souris. La dose moyenne léthale à $37 \%$ de survie, déterminée sur les cellules spermatogoniales a été évaluée à $0,16 \mathrm{~Gy}$ et $0,34 \mathrm{~Gy}$, respectivement avec l'oxine--111 In et du citrate- ${ }^{111}$ In. La dose délivrée par un faisceau de rayon $X$ de référence, pour l'effet correspondant était de 0,67 Gy. Les auteurs ont justifié ce résultat en déterminant, par fractionnement, la localisation sub-cellulaire des deux radiopharmaceutiques au niveau des cellules étudiées. $92 \%$ de l'oxine${ }^{111}$ In était présent dans le noyau, alors que seul $30 \%$ du citrate- ${ }^{111}$ In était localisé dans le noyau. Cette différence de distribution sub-cellulaire de la radioactivité expliquerait les différences de comportement radiotoxicologique des deux radiopharmaceutiques.

Dans un but comparatif, la dose moyenne délivrée au noyau cellulaire par les émissions électroniques a été évaluée suivant le modèle conventionnel et cellulaire (Tab. IV). Cette étude ne tient pas compte des émissions photoniques. Ceci tient 
au fait que la contribution photonique dépend de la taille des organes. La prise en compte de cette contribution peut être réalisée avec les données de la brochure 5 révisée du MIRD (Snyder et al., 1978). La contribution photonique tend à réduire les écarts entre les modèles cellulaire et conventionnelle, et ce d'autant plus, que l'organe cible est plus volumineux. Par exemple, dans le cas du ${ }^{99 \mathrm{~m}} \mathrm{Tc}$, la contribution des émissions électroniques à la dose totale atteint $81,8 \%$ dans le cas d'une localisation thyroïdienne des radiopharmaceutiques technétiés et 44,8\% dans le cas d'une localisation hépatique (Loevinger et al., 1988).

Lorsque la concentration du radiopharmaceutique est identique d'une cellule à l'autre, la comparaison du modèle conventionnel et cellulaire montre que :

- le modèle conventionnel surestime faiblement la dose moyenne due aux électrons qui est délivrée au noyau, dans le cas de localisations cytoplasmiques et membranaires de la radioactivité. Le modèle conventionnel peut en première approximation être utilisé,

- lorsque la radioactivité est concentrée dans le noyau, le modèle conventionnel peut sous-estimer de façon importante la dose électronique moyenne délivrée au noyau cellulaire, il faut alors utiliser le modèle cellulaire pour effectuer les calculs dosimétriques.

Les données biologiques ont également montré, que pour un même tissu, la concentration du radiopharmaceutique peut varier considérablement d'une cellule à l'autre (Gardin et al., 1992). Ce type de situation biologique n'a pas été étudiée ici, mais l'on comprend bien qu'une hétérogénéité de distribution radioactive, ne peut qu'amplifier les écarts de dose entre cellules fortement marquées et faiblement marquées, ainsi qu'entre les évaluations classique et cellulaire. Les cellules fortement marquées voient leurs contributions $D_{\text {self }}^{\text {cel }} \mathrm{s}$ 'accroître de manière linéaire suivant l'accroissement de l'activité cumulée. A l'opposé, les cellules faiblement marquées voient leur contribution $D_{\text {self }}^{\text {cel }}$ décroître suivant $\tilde{A}_{\text {self }}$. Il est là encore utile d'effectuer une dosimétrie cellulaire pour estimer les valeurs et les écarts de dose qui peuvent exister d'un noyau cellulaire à l'autre.

\section{Conclusion}

Cette étude montre qu'en médecine nucléaire diagnostique, la dosimétrie interne conventionnelle ne permet pas toujours d'évaluer les différences de doses absorbées par les noyaux des cellules d'un même tissu. Ceci est le cas, lorsque la radioactivité est intranucléaire ou lors de différences de concentration radioactive d'une cellule à l'autre. Ceci tient au fait que les radio-nucléides utilisés présentent des émissions électroniques de faible énergie, responsable d'une hétérogénéité du dépôt d'énergie à l'échelon cellulaire. La dosimétrie cellulaire peut conduire à une meilleure évaluation des risques radiobiologiques liés à l'administration des radiopharmaceutiques diagnostiques, et à une meilleur radioprotection du patient. 


\section{DOSIMÉTRIE CELLULAIRE EN MÉDECINE NUCLÉAIRE DIAGNOSTIQUE}

\section{Références}

Faraggi M., Gardin I., Bok B. (1990) Microdosimétrie des électrons : évaluation d'une approche analytique et application au cas du technetium 99m. J. Nucl. Med. Biophy., 14, 319-326.

Faraggi M., Gardin I., de Labriolle-Vayley C., Moretti J.L., Bok B. (1994) The influence of tracer localization on the electron dose rate delivered to the cell nucleus by some radionuclides used in diagnostic nuclear medicine procedures. J. Nucl. Med., 35, 113-119.

Gardin I., Colas-Linhart N., Petiet A., Bok B. (1992) Dosimetry at the cellular level of Kupffer cells after technetium-99m-sulphur colloid injection. J. Nucl. Med., 33, 380-384.

Gardin I., Faraggi M., Huc E, Bok B. (1995) Modelling of the relationship between cell dimensions and mean dose delivered to the cell nucleus: application to five radionuclides used in nuclear medicine. Phys. Med. Biol., 40, I001-1014.

Goddu S.M., Rao D.V., Howell R.W. (1994) Multicellular dosimetry for micrometastases: dependence of self-dose versus cross-dose to cell nuclei on type and energy of radiation and subcellular distribution of radionuclides. J. Nucl. Med., 35, 521-530.

Hofer K.G., Harris C.R., Smith J.M. (1975) Radiotoxicity of intracellular ${ }^{67} \mathrm{Ga},{ }^{125} \mathrm{I}$ and ${ }^{3} \mathrm{H}$ : nuclear versus cytoplamic radiation in murine L1210 leukaemia. Int. J. Radiat. Biol., 28, 225-241.

Howell R.W. (1992) Radiations spectra for Auger-electron emitting radionuclides: report $n^{\circ} 2$ of AAPM nuclear medicine task group no. 6, Med. Phys., 19, 1371-1383.

Howell R.W. (1994) The MIRD schema: from organ to cellular dimensions, J. Nucl. Med., 35, 531 533.

Humm J.L., Howell R.W., Rao D.V. (1994) Dosimetry of Auger-electron-emitting radionuclides: report No 3 of AAPM nuclear medicine task group no. 6, Med. Phys., 21, 1901-1915

ICRP (1992) Radiation dose to patients from radiopharmaceuticals, publication 53 de la ICRP. Pergamon press, Oxford.

Kwok C.S., Irfan M., Chan L.B., Prestwich W.V. (1987) Beta dosimetry for radioimmunotherapy of cancer using labeled antibodies, NCI monogr. NCIME, 3, 73-82.

Loevinger R.L., Budinger T.F., Watson E.E. (1988) Dans: MIRD primer for absorbed dose calculations, The Society of Nuclear Medicine, New York.

Makrigiorgos G.M., Adelstein S.J., Kassis A.L. (1989) Limitation of conventional internal dosimetry at the cellular level, J. Nucl. Med., 30, 1856-1864.

Nettleton J.S., Lawson R.S. (1996) Cellular dosimetry of diagnostic radionuclides for spherical and ellipsoidal geometry, Phys. Med. Biol., 41, 1845-1854.

Prestwich W.V., Chan L., Kwok C.S., Wilson B. (1985) Dose point kernels for beta emitting radionucléides. Dans: Proceedings of fourth international radiopharmaceutical dosimetry symposium., 1985, Oak Ridge (Schlafke-Stelson A.T., Watson E.E., Eds). U.S. Department of Energy, DE86010102, Conf-851113, pp. 545-561.

Puncher M.R.B., Blower P. J. (1994) Radionuclide targeting and dosimetry at the microscopic level: the role of microautoradiography, Eur. J. Nucl. Med., 21, 1347-1365.

Rao D.V., Sastry K.S.R., Grimmond H.E., Howell R.W., Govelitz G. F., Lanka V. K., Mylavarapu V. B. (1988) Cytotoxicity of some indium radiopharmaceuticals in mouse testes, J. Nucl. Med., 29. 375-384.

Sastry K.S.R. (1992) Biological effects of the Auger emitter iodine-125: A review. Report N ${ }^{\circ} .1$ of AAPM nuclear medicine task group no. 6, Med. Phys., 19, 1361-1370.

Snyder W.S., Ford M.R., Wamer G.G. (1978) Estimates of specific absorbed fractions for photon sources uniformily distributed in various organs of a heterogeneous phantom. MIRD Pamphlet Number 5 , Revised. The Society of Nuclear Medicine, New York. 